\title{
Formation, destruction and chemical influences of water ice: A review of recent laboratory results
}

\author{
Karin I. Öberg \\ Harvard-Smithsonian Center for Astrophysics \\ 60 Garden St, Cambridge, MA 02138 \\ email: koberg@cfa.harvard.edu
}

\begin{abstract}
Water ice is the dominant constituent of icy grain mantles in the interstellar medium, and as such one of the most abundant species during all stages of star and planet formation. Its formation through atom addition reactions on grain surfaces, its destruction through different desorption channels, and its influence on the chemistry and desorption efficiencies of other species in icy grain mantles have all been the objects of intense study. This contribution reviews our current understanding of these processes, and the laboratory experiments that have been instrumental in establishing the existing paradigm.
\end{abstract}

Keywords. astrochemistry, astrobiology, molecular processes, methods: laboratory, ISM: molecules

\section{Introduction}

Water one of the most abundant volatiles in a diverse set of interstellar environments. Its abundance is often only second to that of $\mathrm{H}_{2}$ and $\mathrm{CO}$. Furthermore water typically dominate the icy mantles that envelope interstellar dust grains in cold and dense interstellar and circumstellar regions, including dense cloud cores, protostellar envelopes and protoplanetary disks mid planes (Öberg et al. 2011, Boogert et al. 2015). Because of its high abundance, and its fundamental connection with prebiotic chemistry, the formation and destruction of water ice (and gas) in space have been the subject of intense study during the past decade (van Dishoeck et al. 2013).

A deep understanding of water ice physics and chemistry is also important to model the formation of complex organic molecules in space, and the division of more volatile species, such as CO, between gas and ice phases. Complex organic molecules (COMs) are proposed to form mainly through grain surface reactions in or on top of icy grain mantles (Herbst \& van Dishoeck 2009, Garrod et al. 2008). In these ices reactive radicals are formed through dissociation of pre-existing ice molecules, especially $\mathrm{CH}_{3} \mathrm{OH}$, that subsequently diffuse and react. Since water is the most abundant ice species it effectively provides the matrix or environment within which this ice chemistry takes place. As such it may regulate the efficiency at which radicals are produced and diffuse. As outlined below, water ice can also inhibit or slow down desorption of other otherwise volatile mantles species through several different mechanisms.

In this proceeding we review the constraints provided by laboratory experiments on the formation, destruction and chemical influences of water ice in interstellar and circumstellar environments (Figure 1). $\S 2$ presents key experiments on water ice formation through $\mathrm{H}$ addition to $\mathrm{O}, \mathrm{O}_{2}$ and $\mathrm{O}_{3}$ on ice surfaces. In $\S 3$ experiments on the main destruction pathways of water, i.e. thermal and non-thermal desorption, are discussed. 
Formation of water icy through grain surface chemistry

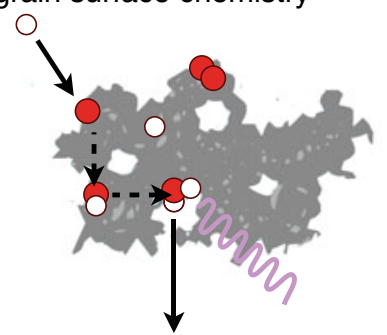

Thermal and non-thermal Desorption of water ice

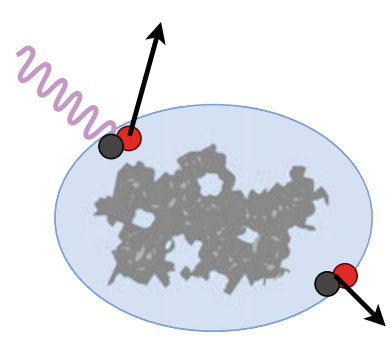

Influence of water ice on desorption of other species
Influence of water ice on formation/destruction of organics

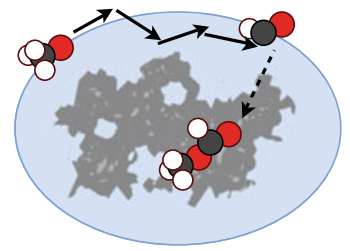

Figure 1. Cartoon summarizing the processes covered in this proceeding.

Experiments aimed at qualifying and quantifying the effects of water on the desorption and chemistry of other ice species are introduced in $\S 4$.

\section{Water ice formation}

Based on theoretical models going back Tielens \& Hagen (1982), water is expected to form on grain surfaces through three different hydrogenation reactions, $\mathrm{H}+\mathrm{O}, \mathrm{H}+\mathrm{O}_{2}$ and $\mathrm{H}+\mathrm{O}_{3}$. All three reactions have been studied in the laboratory during the past few years using high-precision, ultra-high vacuum experiments. Dulieu et al. (2010) used a dual atomic beam experiment to explore water formation through the $\mathrm{O}+\mathrm{H}$ channel and found that, as expected, the reaction does not appear to have any barrier and proceeds with high efficiency, i.e. 40-70\% of the deposited $\mathrm{O}$ atoms are converted into water ice. In their experiments the water ice abundance is not monitored during the formation stage, but rather post formation through temperature programmed desorption experiments. This experimental technique provides limited information on the mechanism of the reaction. The experiments are, however, consistent with the simplest proposed reaction pathway of sequential additions of $\mathrm{H}$ to more or less stationary $\mathrm{O}$ atoms on the surface.

$\mathrm{H}_{2} \mathrm{O}$ ice formation through the $\mathrm{O}_{2}+\mathrm{H}$ and $\mathrm{O}_{3}+\mathrm{H}$ channels have been studied by several groups (Miyauchi et al. 2008, Mokrane et al. 2009, Romanzin et al. 2011, Ioppolo et al. 2010). Based on these experiments water ice can form through both channels under astrochemical relevant conditions. In both cases water formation is accompanied by $\mathrm{H}_{2} \mathrm{O}_{2}$ formation, which distinguishes these channels from the $\mathrm{O}+\mathrm{H}$ one.

By contrast to the $\mathrm{O}+\mathrm{H}$ experiments, some of the $\mathrm{O}_{2}$ and $\mathrm{O}_{3}$ experiments included time resolved monitoring of the forming water and other products (especially $\mathrm{H}_{2} \mathrm{O}_{2}$ ) through infrared spectroscopy, enabling direct constraints on how and when water ice form through these pathways. This is important because both the $\mathrm{O}_{2}+\mathrm{H}$ and $\mathrm{O}_{3}+\mathrm{H}$ channels were expected to proceed through reactions with barriers and thus present temperature dependent formation efficiencies, which needs to be understood to model water formation in different interstellar environments. Surprisingly, Ioppolo et al. (2010) did not find such a temperature dependent rate for $\mathrm{O}_{2}+\mathrm{H}$. This result was explained by competition between diffusion and reaction, where diffusion and reactions present comparable barriers. Ioppolo et al. (2010) did find, however, an increasing formation efficiency with increasing temperature, which can be explained by an increasing penetration depth of $\mathrm{H}$ atoms into the ice at higher temperatures where molecules are more mobile, stressing the importance of ice morphology and the role of the ice matrix for ice chemistry. 


\section{Water ice desorption}

Icy grain mantles, including water ice, can desorb (sublimate) thermally or nonthermally. Thermal desorption of astrochemical relevant water ices was well characterized more than a decade ago by Fraser et al. (2001). Recent efforts have instead focused on different kinds of non-thermal desorption, especially UV induced photodesorption and chemical desorption following water formation. Photodesorption of water ice was first quantified by Westley et al. (1995) revealing a high efficiency $\left(\sim 10^{-3}\right.$ molecules desorbed per incident UV photon). This high yield was confirmed by (Öberg et al. 2009), who also uncovered new aspects of the desorption mechanism including 1 . an increasing number of desorption-active layers with increasing temperature, and 2. that $\mathrm{H}_{2} \mathrm{O}$ is desorbed both as $\mathrm{OH}$ and as $\mathrm{H}_{2} \mathrm{O}$. The mechanism of the desorption process was further explored theoretically by Anderson \& van Dishoeck (2008) and experimentally by Yabushita et al. (2009) and Hama et al. (2010), who demonstrated that the most important desorption mechanism is through the 'kick-out' of a water surface molecule by an energetic $\mathrm{H}$ atom produce through photodissociation of a sub-surface water molecule, i.e. a rather indirect photodesorption mechanism.

Chemical desorption takes place when some of the energy released from chemical bond formation (e.g. forming the $\mathrm{O}-\mathrm{H}$ bond in water) is channeled into desorption of the newly formed molecule. This desorption channel is frequently invoked in astrochemical models, but has been very challenging to quantify experimentally or theoretically. A recent laboratory experiment employing the temperature programmed desorption method found a very high chemical desorption efficiency for water ice, i.e. a $>90 \%$ desorption efficiency, implying that the vast majority of formed water molecules are desorbed into the gas phase (Dulieu et al. 2013). This value awaits confirmation and would indeed be very surprising if true for interstellar ices, since it would efficiently inhibited ice build-up, which contrary to what is observed.

\section{Influence of water ice on chemistry and desorption of other ices}

Figure 2 illustrates how water ice can slow down or inhibit the desorption of other ice molecules, substantially changing their relative gas and ice abundance compared to a scenario where water is absent. Water ice can physically trap molecules (i.e. no clathrate formation is required) both when the other molecule (e.g. CO) is deposited underneath the water ice (Fayolle et al. 2011a), and on top of porous water ice. In the latter case entrapment proceeds through pore collapse (Collings et al. 2003). The presence of water ice also increases the desorption temperature for some molecules, including the astrochemical important $\mathrm{CO}$ and $\mathrm{N}_{2}$ (Collings et al. 2004). These enhancements can be large. Sub-Monolayers of $\mathrm{CO}$ and $\mathrm{N}_{2}$ deposited on top of porous water ice presents almost a factor of two higher desorption energy compared to pure $\mathrm{CO}$ and $\mathrm{N}_{2}$ ice (Fayolle et al. private communication). Water ice can thus generally inhibit thermal desorption of other ice molecules, but for most molecules the effects of water have yet to be quantified.

Water has an equally important effect on non-thermal desorption yields. Bertin et al. (2012) found that $\mathrm{CO}$ ice UV photodesorption is almost completely inhibited when $\mathrm{CO}$ is deposited on top of water ice. This is in starch contrast with pure CO photodesorption, which is highly efficient with yields of $10^{-3}-10^{-2}$ per incident photon (Fayolle et al. 2011b). The proposed explanation is that CO photodesorbs indirectly through a kick from an excited, sub-surface $\mathrm{CO}$ molecule and thus requires a sub-surface layer of $\mathrm{CO}$ molecules to efficiently photodesorb. 


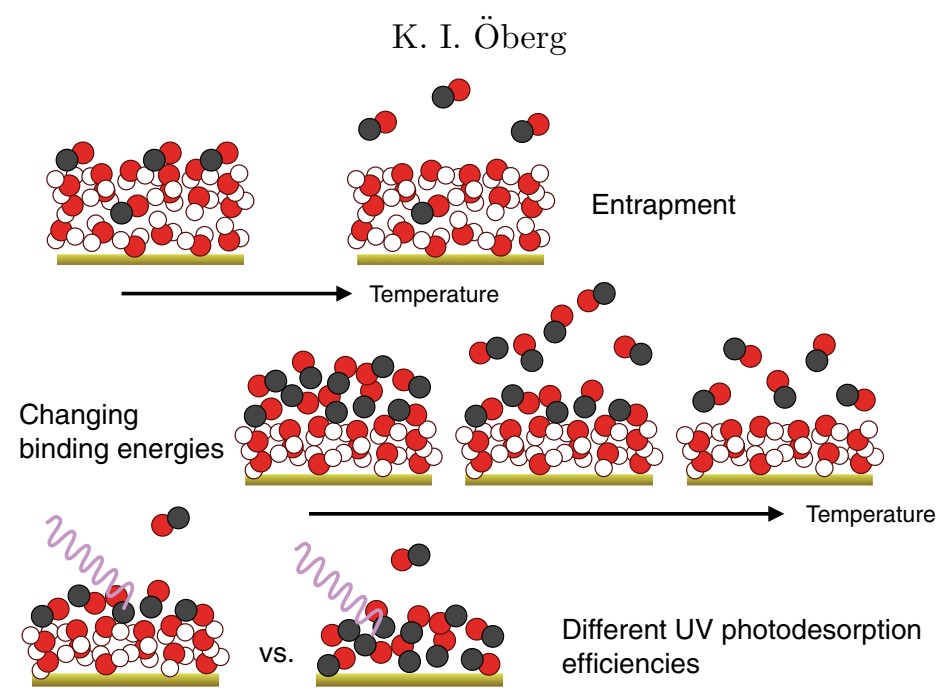

Figure 2. Illustration of how water can interfere with the desorption of other ice molecules through entrapment, through formation of stronger intermolecular bonds compared to pure ices, and through inhibition of non-thermal desorption including photodesorption.

Finally, water ice affects/inhibits the formation efficiencies of complex organic molecules. First, photodissociation of molecules, the process through which COM reactants are formed in ices, is reduced when water ice is present (Öberg et al. 2010). For example the photodissociation rate of $\mathrm{CH}_{4}$ into $\mathrm{CH}_{3}$ and $\mathrm{CH}_{2}$ is an order of magnitude higher in pure $\mathrm{CH}_{4}$ ice compared to a water dominated ice. The water is likely 'caging' the $\mathrm{CH}_{4}$ molecules resulting in rapid radical recombination following dissociation, reducing the effective dissociation rate. Second, the presence of water changes the diffusion environment for radicals and molecules in the ice, which slows down a diffusion limited chemistry such as the radical-radical reactions proposed to produce COMs in the ISM. Attempts to quantify diffusion in $\mathrm{H}_{2} \mathrm{O}$ ice is currently underway (e.g. Lauck et al. 2015).

In summary, $\mathrm{H}_{2} \mathrm{O}$ ice affects the composition of icy grain mantles in profound ways that are only beginning to be be understood, much less quantified. A concerted laboratory and theoretical effort on this subject seems appropriate considering that icy grain mantles are the proposed chemical factories of the ISM, and their compositions are thus key to our understanding of the chemical evolution during star and planet formation.

\section{References}

Andersson, S. \& van Dishoeck, E. F. 2008, A\&A 491, 907

Bertin, M., Fayolle, E. C, Romanzin, C., Öberg, K. I., Michaut, X., Moudens, A., Philippe, L., Jeseck, P., Linnartz, H., \& Fillion, J.-H. 2012, PCCP 14, 9929

Boogert, A. C. A., Gerakines, P. A, \& Whittet, D. C. B. 2015, ARA\& A 53, 541

Collings, M. P., Dever, J. W., Fraser, H. J., Mccoustra, M. R. S, \& Williams, D. A. 2003, ApJ 583,1058

Collings, M. P., Anderson, M. A., Chen, R., Dever, J. W., Viti, S., Williams, D. A., \& Mccoustra, M. R. S. 2004, MNRAS 354, 1133

Dulieu, F., Amiaud, L., Congiu, E., Fillion, J.-H., Matar, E., Momeni, A., Pirronello, V., \& Lemaire, J. L. 2010, A\& $A$ 512, 30

Dulieu, F., Congiu, E., Noble, J., Baouche, S., Chaabouni, H., Moudens, A., Minissale, M., \& Cazaux, S. 2013, Nature Sci. Rep. 3, 1338

Fayolle, E. C, Öberg, K. I, Cuppen, H. M., Visser, R., \& Linnartz, H. 2011a A $\& A$ 529, 74 
Fayolle, E. C., Bertin, M., Romanzin, C., Michaut, X., Öberg, K. I., Linnartz, H., \& Fillion, J.-H. 2011b, $A p J$ (Letters) 739, L36

Fraser, H. J., Collings, M. P., Mccoustra, M. R. S., \& Williams, D. A., 2001, MNRAS 327, 1165

Garrod, R. T., Weaver, S. L. W., \& Herbst, E. 2008, ApJ 682, 283

Hama, T., Yokoyama, M., Yabushita, A., Kawasaki, M., Andersson, S., Western, C. M., Ashfold, M. N. R., Dixon, R. N., \& Watanabe, N. 2010, JCP 132, 4508

Herbst, E. \& van Dishoeck, E. F. 2009, ARA\& A 47, 427

Ioppolo, S., Cuppen, H. M., Romanzin, C., van Dishoeck, E. F., \& Linnartz, H., 2010, PCCP 12,12065

Lauck, T., Karssemeijer, L., Shulenberger, K., Rajappan, M., Öberg, K. I., \& Cuppen, H. M. 2015, ApJ 801, 118

Miyauchi, N. Hidaka, H. Chigai, T., Nagaoka, A., Watanabe, N., \& Kouchi, A. 2008 Chem. Phys. Lett. 456, 27

Mokrane, H., Chaabouni, H., Accolla, M., Congiu, E., Dulieu, F., Chehrouri, M., \& Lemaire, J. L. 2009, ApJ (Letters) 705, 195

Öberg, K. I., Linnartz, H., Visser, R., \& van Dishoeck, E. F. 2009, ApJ 693, 1209

Öberg, K. I., Van Dishoeck, E. F., Linnartz, H., \& Andersson, S. 2010, ApJ 718, 832

Öberg, K. I., Boogert, A. C. A., Pontoppidan, K. M., van den Broek, S., van Dishoeck, E. F., Bottinelli, S., Blake, G. A., \& Evans, N. J., II 2011, ApJ 740, 109.

Romanzin, C, Ioppolo, S, Cuppen, H. M., van Dishoeck, E. F., \& Linnartz, H. 2011, J. of Chem. Phys. 134, 4504

Tielens, A. G. G. M, \& Hagen, W. 1982 A\&A 114, 245

van Dishoeck, E. F., Herbst, E., \& Neufeld, D. A., 2013, Chem. Rev. 113, 9043

Westley, M. S, Baragiola, R. A, Johnson, R. E, \& Baratta, G. A . 1995, Nature 373, 405

Yabushita, A., Hama, T., Yokoyama, M., Kawasaki, M., Andersson, S., Dixon, R. N., Ashfold, M. N. R., \& Watanabe, N. 2009, ApJ (Letters) 699, L80 